8th Alexander Friedmann International Seminar

on Gravitation and Cosmology

International Journal of Modern Physics: Conference Series

Vol. 3 (2011) 408-416

(C) World Scientific Publishing Company

DOI: 10.1142/S2010194511000924

\title{
BLACK HOLES COLLISION IN GENERAL ROBINSON-TRAUTMAN SPACETIMES: WAVE FORMS AND THE EFFICIENCY OF THE GRAVITATIONAL WAVE EXTRACTION
}

\author{
H. P. DE OLIVEIRA and E. L. RODRIGUES \\ Universidade do Estado do Rio de Janeiro \\ Instituto de Física - Departamento de Física Teórica \\ CEP 20550-013 Rio de Janeiro, RJ, Brazil \\ hp.deoliveira@pq.cnpq.br, elrodrigues@uerj.br
}

Received 15 June 2011

Revised 5 July 2011

\begin{abstract}
We analyze the non-frontal collisions of two Schwarzschild black holes in the realm of general Robinson-Trautman spacetimes using a numerical code based on spectral methods. In this process, two black holes collide and form a single black hole while a certain amount of the initial mass is carried away by gravitational waves. We determined the forms of the gravitational waves and the efficiency of this process for frontal and nonfrontal collisions. We found numerical evidence that the distribution of mass qloss can be described by a function typically used in nonextensive statistics.
\end{abstract}

Keywords: Numerical relativity; black holes collision; gravitational waves.

PACS numbers: 04.25.dg , 04.30.Db , 02.70.Hm

\section{Introduction}

The use of numerical techniques to solve Einstein's field equations is a very promising strategy for dealing with problems of astrophysical interest such as gravitational collapse and formation of black holes, coalescence of binary systems, the collision of black holes, etc. One of the most notable predictions of General Relativity the emission of gravitational waves - is the common feature in all these processes. Undoubtedly, detailed knowledge of how much system mass is converted into gravitational waves and the gravitational wave templates are of fundamental importance toward the efforts to a direct detection of gravitational radiation with ground and space based observatories. ${ }^{1}$

In this context, using an efficient numerical code for solving the field equations of general Robinson-Trautmann spacetimes ${ }^{2}$ based on spectral methods, ${ }^{3}$ we exhibit the initial results for the wave forms and efficiency of the gravitational wave extraction associated with the non-frontal collision of two Schwarzschild black holes.

The paper is organized as follows. In Sec. 2 we present some basic aspects of general Robinson-Trautman spacetimes. In Sec. 3 we consider an initial data describing 
the collision of two Schwarzchild black holes in three situations: orthogonal, oblique and head-on collisions. We show some examples of the gravitational wave forms and the efficiency of the gravitational wave extraction evaluated after evolving the spacetime. Finally, in Sec. 4 we present a summary of our results and conclusions.

\section{General Robinson-Trautman Spacetimes}

Robinson-Trautman (RT) metrics are solutions of vacuum Einsteins equations representing an isolated gravitational radiating system. The general RT metric can be expressed as, ${ }^{4}$

$$
d s^{2}=\left(\lambda-\frac{2 m_{0}}{r}-2 r \frac{\dot{P}}{P}\right) d u^{2}+2 d u d r-\frac{r^{2}}{P^{2}}\left(d \theta^{2}+\sin ^{2} \theta d \phi^{2}\right),
$$

where $u$ is a null coordinate such that $u=$ constant denotes null hypersurfaces generated by the rays of the gravitational field and that foliates the spacetime globally; $r$ is an affine parameter defined along the null geodesics determined by the vector $\partial / \partial r$ and $m_{0}$ is a constant. The metric function $P$ depends on the coordinates $u, \theta, \phi$. In the above expression an overdot indicates a derivative with respect to $u, m_{0}$ is a constant and the function $\lambda$ is identified as the Gaussian curvature of the 2-surfaces. In the particular case of axial symmetry, the metric functions do not depend on $\phi$. Three important aspects of Robinson-Trautman spacetimes are worth mentioning: they are asymptotically flat, they admit the presence of gravitational waves and the end asymptotic state of these spacetimes is the Schwarzschild solution.

The vacuum Einstein's equations for the RT spacetimes reduce to,

$$
\begin{aligned}
& \lambda=P^{2}+\frac{P^{2}}{\sin \theta}\left(\sin \theta \frac{P_{, \theta}}{P}\right)_{, \theta}+\frac{P^{2}}{\sin ^{2} \theta}\left(\frac{P_{, \phi}}{P}\right)_{, \phi}, \\
& 12 m_{0} \frac{\dot{P}}{P}+P^{2}\left(\frac{\left(\lambda_{, \theta} \sin \theta\right)_{, \theta}}{\sin \theta}+\frac{\lambda_{, \phi \phi}}{\sin ^{2} \theta}\right)=0,
\end{aligned}
$$

where the subscripts $\theta, \phi$ denote derivatives with respect to the angles $\theta$ and $\phi$, respectively. The structure of the field equations is typical of the characteristic evolution scheme: Eq. (2) is the hypersurface equation that defines $\lambda(u, \theta, \phi)$ and Eq. (3), known as the RT equation, governs the dynamics of the gravitational field. In other words, from the initial data $P\left(u_{0}, \theta, \phi\right)$, the hypersurface equation determines $\lambda\left(u_{0}, \theta, \phi\right)$ and the RT equation allows us to evolve the initial data.

The full evolution of the field equations (2) and (3) constitutes a valid and valuable framework for a detailed analysis of the wave forms and the efficiency of the gravitational wave extraction connected with the formation of black holes in non-axisymmetric spacetimes. Some of these aspects were addressed in Refs. 5-11 in the context of axial symmetry after the development of a numerical code for integrating the field equations. ${ }^{12}$ 
In Ref. 2 we presented an efficient numerical code based on spectral methods to integrate the field equations of general Robinson-Trautmann spacetimes. The most natural basis functions for the spectral expansion of the metric functions are spherical harmonics. These expansions are given by, ${ }^{2}$

$$
\begin{aligned}
& P_{N}(u, \theta, \phi)=\sum_{k=0}^{N} \sum_{l=-k}^{l=k} a_{k l}(u) Y_{k l}(\theta, \phi), \\
& \lambda_{N}(u, \theta, \phi)=\sum_{k=0}^{N} \sum_{l=-k}^{l=k} b_{k l}(u) Y_{k l}(\theta, \phi),
\end{aligned}
$$

where $Y_{k j}(\theta, \phi)$ are the spherical harmonics, $a_{k l}(u)$ and $b_{k l}(u)$ are the unknown modes and $N$ is the truncation order that dictates the number of independent modes. Using the values of appropriate combinations of the metric functions at the collocation points, we have managed to reduce expression swell when the number of spherical harmonics increases. Our numerical code runs with relatively little computational resources and the code tests have shown excellent accuracy and convergence.

Cornish $^{13}$ has shown that the asymptotic $(u \rightarrow \infty)$ expression for the metric function $P$ is expressed as,

$$
P_{\text {final }}(x, \phi)=P_{0}\left[\cosh \gamma+a x \sinh \gamma+\sinh \gamma \sqrt{1-x^{2}}(b \cos \phi+c \sin \phi)\right],
$$

where $x=\cos \theta$. This solution describes a black hole boosted in an arbitrary direction with respect to an asymptotic observer with velocity parameter $v=\tanh \gamma$. The parameters $a, b$ and $c$ satisfy the relation,

$$
a^{2}+b^{2}+c^{2}=1
$$

as a consequence of Eq. (2). Notice that $b=c=0$ implies $a= \pm 1$, recovering the axially boosted black hole, ${ }^{14}$ where the signs \pm indicate opposing directions of movement along the $z$-axis. In the general case, the black hole is moving with respect to an inertial observer in an arbitrary direction determined by $a, b$ and $c$, which can be identified as the direction cosines. ${ }^{10}$ In this case, the total mass-energy of the black hole is given by,

$$
E_{\mathrm{BH}}=\frac{m_{0}}{4 \pi} \int_{0}^{2 \pi} \int_{-1}^{1} \frac{d \phi d x}{P_{\text {final }}^{3}(x, \phi)}=m_{\text {rest }} \cosh \gamma,
$$

where $m_{\text {rest }}=m_{0} P_{0}^{-3}$ is the rest mass of the boosted black hole. The velocity of the hole is thus given by $\tanh \gamma$ as in the axisymmetric case.

One of the most important aspects of RT spacetimes is the presence of gravitational waves. ${ }^{4}$ As discussed in Refs. 5, 7, 8, the characterization of gravitational waves in RT spacetimes is based on the Peeling theorem, ${ }^{15}$ for which the Weyl tensor is expressed as $C_{A B C D} \sim N_{A B C D} / r$ at large $r$ characterizing the wave zone. The 
relevant components of the Weyl tensor with respect to a semi-null tetrad basis ${ }^{5}$ are $C_{0303}=-C_{0202}=-D(u, x, \phi) / r+\mathcal{O}\left(1 / r^{2}\right)$, where

$$
D(u, x, \phi)=\frac{P^{2}}{2} \frac{\partial}{\partial u}\left[\left(1-x^{2}\right) \frac{P_{, x x}}{P}-\frac{1}{\left(1-x^{2}\right)} \frac{P_{, \phi \phi}}{P}\right] .
$$

This function gives the time and angular dependence of the gravitational wave amplitude within the wave zone.

\section{Black Holes Collision: Wave Forms and Efficiency of Gravitational Wave Extraction}

We studied the efficiency and waveforms of gravitational waves emission of the non-frontal collision of two Schwarzschild black holes initially with the same speed $v=\tanh \gamma$ described by the following initial data: ${ }^{2}$

$$
P\left(u_{0}, \theta, \phi\right)=\left(\frac{1}{\sqrt{P_{+}(\theta, \phi)}}+\frac{1}{\sqrt{P_{-}(\theta, \phi)}}\right)^{-2},
$$

where $P_{ \pm}=P_{ \pm}^{(0)}\left[\cosh \gamma+a_{ \pm} \sinh \gamma \cos \theta+\sinh \gamma \sin \theta\left(b_{ \pm} \cos \phi+c_{ \pm} \sin \phi\right)\right]$, with $a_{ \pm}^{2}+b_{ \pm}^{2}+c_{ \pm}^{2}=1$. Note that the particular case $b_{ \pm}=c_{ \pm}=0, a_{ \pm}= \pm 1$ reduces to the initial data for two initially boosted Scharwzschild black holes with opposite velocities tanh $\gamma$ along the symmetry axis. ${ }^{7}$ By conveniently setting these parameters we can study three situations illustrated by Fig. 1, that is, two non-frontal collisions and one frontal collision. In all situations the initial motion of the first black hole is along the $z$-axis, with $a_{+}=1, b_{+}=c_{+}=0$, whereas the second black hole has in turn (i) $a_{-}=b_{-}=0, c_{-}=1$ corresponding to a direction orthogonal to the $z$-axis, (ii) $a_{-}=-\sqrt{2} / 2, b_{-}=0, c_{-}=-\sqrt{2} / 2$, and (iii) $a_{-}=-1, b_{-}=c_{-}=0$ for a head-on collision. In all numerical experiments, $P_{+}^{(0)}=1$ and $P_{-}^{(0)}$ is a free parameter that defines the ratio $\eta$ between the initial rest masses of the two black holes,

$$
\eta=\frac{m_{\mathrm{rest}}^{-}}{m_{\mathrm{rest}}^{+}}=\left(P_{-}^{(0)}\right)^{-3}
$$
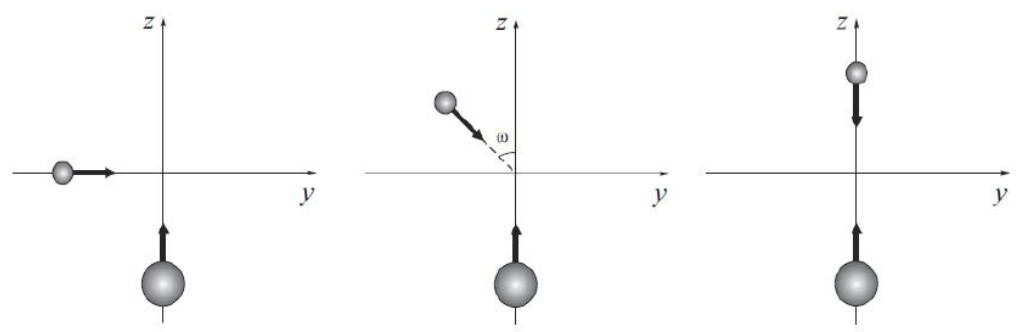

Fig. 1. Illustration of the three situations representing (from left to right) orthogonal, oblique and head-on collisions of two Schwarzschild black holes. 


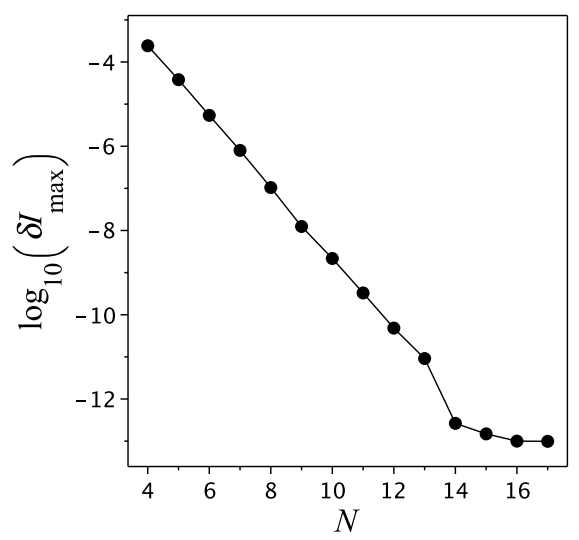

Fig. 2. Log-linear plot of the maximum relative error $\delta I$ after evolving the field equations with the initial data $(10)$ for the oblique collision with $P_{-}^{(0)} \simeq 6.3\left(P_{+}^{(0)}=1\right)$ and $\gamma=0.7$. Notice that rapid decay of this error when the $N$ increases is a clear numerical evidence of the spectral convergence of the code.

Before proceeding with our results on the angular pattern of gravitational waves, we judge an important to show briefly the accuracy and convergence of the numerical code. The test consists in checking the conserved quantity ${ }^{16}$

$$
I=\frac{1}{4 \pi} \int_{0}^{2 \pi} \int_{-1}^{1} P^{-2} d x d \phi .
$$

The initial data (10) determines the exact value $I=I_{\text {exact }}$ that is maintained until the asymptotic state is reached. On the other hand, by integrating numerically the field equations we can determine the approximate value of the above constant, $I_{\text {numer }}$, at each instant and comparing it with the exact value. In Fig. 2 we depict exponential decay of the maximum relative error $\delta I=\left|I_{\text {exact }}-I_{\text {numer }}\right| / I_{\text {exact }}$ with increasing $N$ attesting the accuracy and convergence of the code. and comments)

In Figs. 3 and 4 we show the structure and evolution of the angular pattern of gravitational waves, displaying a sequence of three dimensional polar plots of
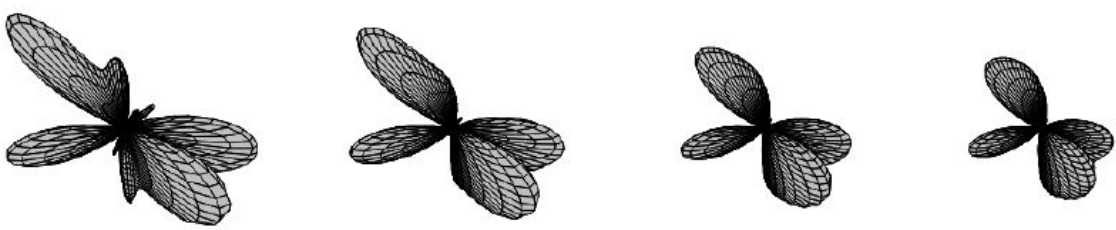

Fig. 3. Sequence of three dimensional polar plots of $D(u, \theta, \phi)$ corresponding to the orthogonal collision of two Schwarzschild black holes evaluated at $u=0.5,1.5,3.5,5.5$. The lobes determine the directions of maximum magnitude of the emitted gravitational waves, and the lack of symmetry of the angular pattern indicates that the resulting black hole might be moving with respect to an asymptotic observer. 

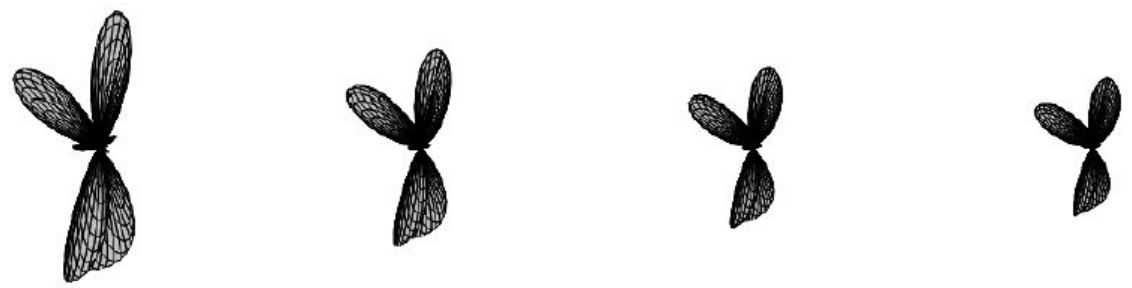

Fig. 4. Sequence of three dimensional polar plots of $D(u, \theta, \phi)$ corresponding to the oblique collision of two Schwarzschild black holes evaluated at $u=0.0,1.0,2.0,3.0$. Again, as in Fig. 3 the angular pattern is characterized by an antisymmetric structure of lobes, but in a distinct configuration. The final black hole is described by the stationary solution (6).

$D(u, \theta, \phi)$. The sequences correspond to the orthogonal and oblique collision of two Schwarzschild black holes [cf. Eq. (10)], respectively, where $D(u, \theta, \phi)$ is evaluated at several times. The non-symmetric pattern of $D$ indicates the existence of a net flux of momentum carried away by gravitational waves. In those cases, due to the conservation of momentum-energy, the end state is a boosted black hole described by Eq. (6). The boost parameter was fixed as $\gamma=0.9$ and $\eta=0.1$.

As we can see in Fig. 5, that represent the evolution of $D$ for the oblique collision projected on a fixed plane $x=0$ and $x=0.3$, respectively, the angular pattern is dominated by lobes which indicate the directions of maximum magnitude of the gravitational wave emission. Note that the two dominant lobes open as the black hole is decelerated, forming a bremsstrahlung-like pattern.

Another important result is the efficiency of gravitational wave emission that measures the amount of mass-energy extracted during the evolution of RT spacetime
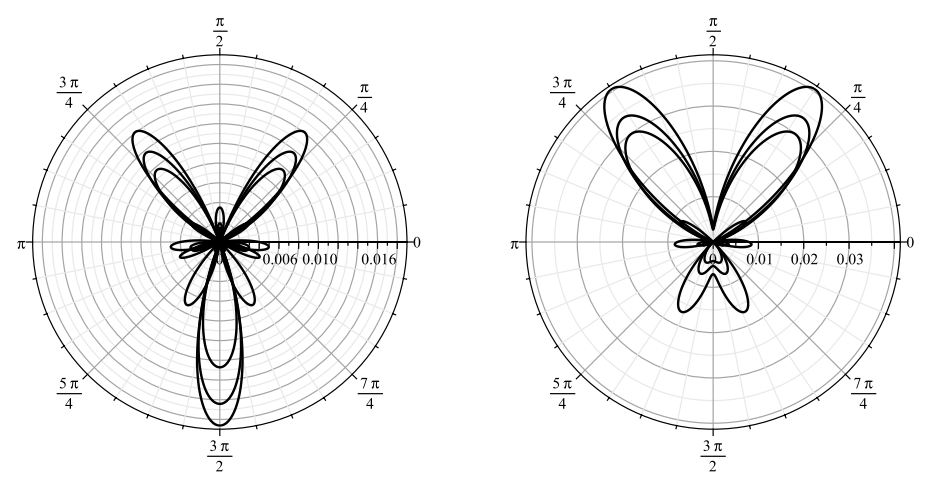

Fig. 5. Illustration of the sequence of polar plots of $D$ projected on the planes $x=0(u=$ $0.02,0.10,0.26)$ and $x=0.3(u=0.02,0.10,0.18)$ for the oblique collision. The angular pattern is dominated by lobes which indicate the directions of maximum magnitude of the gravitational wave emission. Note that the two dominant lobes open as the black hole decelerated, forming a bremsstrahlung-like pattern. 
Table 1. Efficiency and the final boost of the remnant black hole for several values of $\eta$.

\begin{tabular}{|c|c|c|c|c|c|c|}
\hline & \multicolumn{2}{|c|}{ Orthogonal } & \multicolumn{2}{c|}{ Oblique } & \multicolumn{2}{c|}{ Head-on } \\
\hline$\eta$ & $v_{f}$ & $\Delta(\%)$ & $v_{f}$ & $\Delta(\%)$ & $v_{f}$ & $\Delta(\%)$ \\
\hline 0.001 & 0.601 & 0.608 & 0.537 & 1.23 & 0.514 & 1.473 \\
\hline 0.008 & 0.564 & 0.911 & 0.456 & 1.97 & 0.411 & 2.428 \\
\hline 0.125 & 0.520 & 1.307 & 0.327 & 3.019 & 0.202 & 3.797 \\
\hline 0.296 & 0.511 & 1.384 & 0.294 & 3.233 & 0.121 & 4.084 \\
\hline
\end{tabular}

with the eventual formation of a black hole. It is expressed as

$$
\Delta=\frac{E_{\mathrm{I}}-E_{\mathrm{BH}}}{E_{\mathrm{I}}}=1-\frac{m_{\mathrm{rest}} \cosh \gamma_{f}}{E_{\mathrm{I}}},
$$

where $E_{\mathrm{I}}$ is the mass-energy of the initial system, $E_{\mathrm{BH}}=m_{\text {rest }} \cosh \gamma_{f}$ is the energy of the remnant black hole and $\gamma_{f}$ is the final boost parameter.

In Table 1 we summarize the results for several values of $\eta$, where we fixed $\gamma=0.9$, which corresponds to the initial relativistic velocity $v \approx 0.716$. Note that the frontal collision is the most efficient in terms of carrying away energy by gravitational waves. In line with numerical simulations based on more realistic scenarios, the efficiency of the extraction of energy via gravitational waves never exceeds a few percent of the initial energy.

We also obtained the efficiency of gravitational waves emission for several values of the boost parameter, $\gamma=0.5,0.6,0.7,0.8$ for which the velocities range between approximately $138,000 \mathrm{~km} / \mathrm{s}$ and $199,000 \mathrm{~km} / \mathrm{s}$. For each value of the boost parameter, the field equations were evolved for frontal and non-frontal collisions until the formation of the black hole end state. Those results can be seen in Fig. 6. Note that the maximum value of $\Delta$ occurs for equal initial rest masses, or $\eta=1$.

Following our previous works ${ }^{5-9}$ based on non-extensive relations inspired by the Tsallis statistics, ${ }^{19}$ we have found that a q-Weibull distribution ${ }^{20}$ fits the numerical data $(\eta, \Delta)$ (cf. Fig. 6). The q-Weibull distribution has been used to describe a variety of statistical systems and is given by ${ }^{20}$

$$
\Delta=\Delta_{0} \eta^{\nu-1}\left[1+(q-1)\left(\frac{\eta}{\eta_{0}}\right)^{\nu}\right]^{\frac{1}{1-q}},
$$

where $q$ and $\nu$ are parameters that describe the power-law regimes for very large and small values of $\eta$. In Fig. 6 we show graphs of the numerical data (circles) corresponding to the head-on and oblique collisions, and a continuous line representing the function (14). We have found that $q$ has the same value, $q \approx 2.15$ irrespective of the type of collision or the initial velocities. The values of $\nu$ vary slightly with respect to the velocity: for example in the case of the oblique collision, $\nu=2.655,2.705,2.77,2.83$ for the previously cited velocities.

It is surprising that the non-extensive function (14) with a fixed value of $q$ describes the distribution of the mass extracted by gravitational waves. Recently we have shown that a q-exponential distribution relates the fraction of absorbed 

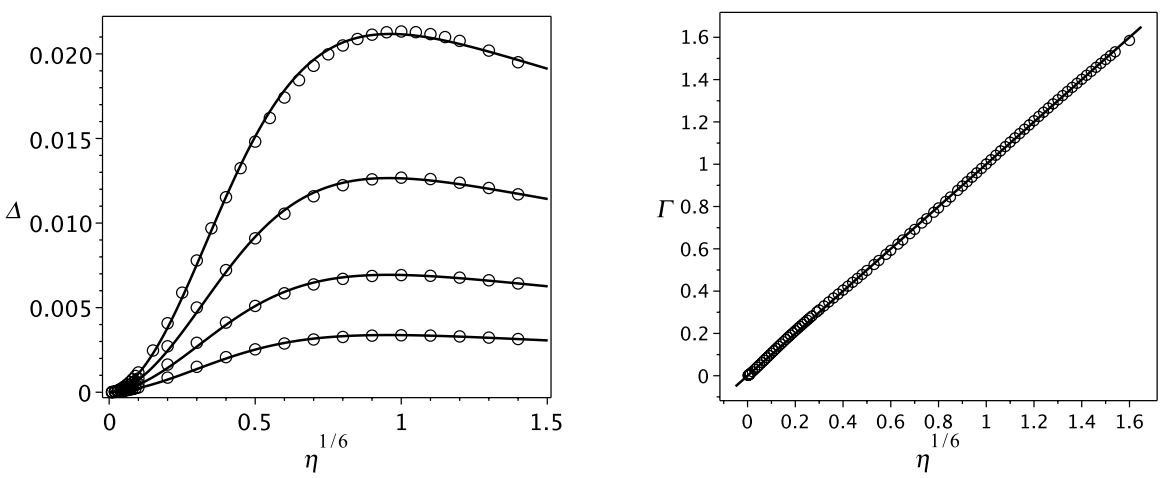

Fig. 6. Plots of the numerical data (circles) and the q-Weibull distribution (14) corresponding to the oblique collision with $\gamma=0.5,0.6,0.7,0.8$ (from bottom to top). Note that the maximum value of $\Delta$ occurs for equal initial rest masses, or $\eta=1$. The graph on the right corresponds to the head-on collison with $\gamma=0.7$ and shows the function $\Gamma=\left[\frac{\Delta}{\Delta_{0}}\left(1+(q-1)\left(\frac{\eta}{\eta_{0}}\right)^{\nu}\right)^{\frac{1}{1-q}}\right]^{\frac{1}{\nu-1}}$ versus $\eta$. The straight continuous line has been calculated exactly from (14) while the curves passing through the circles were obtained using a best fit.

mass to form a black hole with the amplitude of the initial scalar field packet in the case of spherically symmetric collapse. ${ }^{18}$

\section{Discussion}

In this paper we have studied numerically the dynamics of general RobinsonTrautman geometries.

We point out that a thorough study of the non-frontal collision of two Schwarzschild black holes in the realm of RT spacetimes is a subject for further investigation. The initial data describing a non-frontal collision of two Schwarzschild black holes with the same speed, given by Eq. (10), generalizes the initial data established for the head-on collision presented in Ref. 7. The numerical integration of the field equations (2) and (3) provides a useful theoretical probe for studying the consequences of the non-frontal collision of two black holes. ${ }^{17}$ We have presented a visualization of the angular pattern of the gravitational radiation defined in the wave zone [cf. Eq. (9)], whose structure depends on the initial data.

The second part of this work concentrates on the efficiency of the conversion of the initial mass to gravitational waves. We confirmed that a head-on collision is the most efficient source for extracting gravitational radiation, although obviously being a rarer astrophysical event than a non-frontal collision. The evolution of the apparent horizon would also be worth investigating.

Finally, as a possible consequence of the interplay between gravitation and thermodynamics, we found numerical evidence that the distribution of mass loss can be described by a function typically used in nonextensive statistics. 


\section{Acknowledgments}

The authors acknowledge the financial support of the Brazilian agencies CNPq, CAPES and FAPERJ.

\section{References}

1. B. S. Sathyaprakash and B. F. Schutz, Liv. Rev. Relativity 12, 2 (2009); B. F. Schutz and F. Ricci, Gravitational waves, sources and detectors, arXiv:1005.4735v1.

2. H. P. de Oliveira, E. L. Rodrigues and J. E. F. Skea, Numerical evolution of general Robinson-Trautman spacetimes: Code tests, wave forms and the efficiency of the gravitational wave extraction, arXiv:1107.0694, to appear in Phys. Rev. D (2011).

3. $\mathrm{P}$ Grandclément and J. Novak, Liv. Rev. Relativity 12, 1 (2009); http://www.livingreviews.org/lrr-20091 .

4. I. Robinson and A. Trautman, Phys. Rev. Lett. 4, 431 (1960); Proc. Roy. Soc. A 265, 463 (1962).

5. H. P. de Oliveira and I. Damião Soares, Phys. Rev. D 70, 084041 (2004).

6. H. P. de Oliveira and I. Damião Soares, Phys. Rev. D 71, 124034 (2005).

7. R. F. Aranha, H. P. de Oliveira, I. D. Soares and E. V. Tonini, Int. J. Mod. Phys. D 17, 1 (2008).

8. H. P. de Oliveira, I. Damião Soares and E. V. Tonini, Phys. Rev. D 78, 044016 (2008).

9. H. P. de Oliveira and E. L. Rodrigues, Class. Quant. Grav. 25, 205020 (2008).

10. R. P. Macedo and A. Saa, Phys. Rev. D 78, 104025 (2008).

11. L. Rezzola, R. P. Macedo and J. L. Jaramillo, Phys. Rev. Lett. 104, 221101 (2010).

12. H. P. de Oliveira, E. L. Rodrigues, I. Damião Soares and E. V. Tonini, Int. J. Mod. Phys. C 18, 1839 (2007).

13. F. H. J. Cornish, Class. Quant. Grav. 17, 3945 (2000).

14. H. Bondi, M. G. J. van der Berg and A. W. K. Metzner, Proc. Roy. Soc. A 269, 21 (1962).

15. R. Sachs and P. G. Bergmann, Phys. Rev. 112, 674 (1958); R. Sachs, Proc. Roy. Soc. A 264, 309 (1961); Proc. Roy. Soc. A 270, 103 (1962); E. T. Newman and R. Penrose, J. Math. Phys. 3, 566 (1962).

16. E. W. M. Chow and A. W. C. Lun, J. Austral. Math. Soc. B 41, 217 (1999).

17. O. M. Moreschi and S. Dain, Phys. Rev. D 53, R1745 (1996); O. M. Moreschi, A. Perez and L. Lehner, Phys. Rev. D 66, 104017 (2002).

18. H. P. de Oliveira, E. L. Rodrigues, and J. E. Skea, Phys. Rev. D 82, 104023 (2010).

19. C. Tsallis, J. Statist. Phys. 52, 479 (1988).

20. S. Picoli Jr., R. S. Mendes, L. C. Malacarne and R. P. B. Santos, Braz. J. Phys. 39, 468 (2009). 